\section{SOCIAL CLASS DIFFERENCES IN ANXIETY AND DEPRESSION ACROSS THE LIFE-COURSE: EVIDENCE FROM THREE COHORTS IN THE WEST OF SCOTLAND}

MJ Green, M Benzeval. Medical Research Council Social and Public Health Sciences Unit, Glasgow, UK

\section{doi:10.1136/jech.2009.096701s}

Background: Studies of social inequalities in common mental disorders - anxiety and depression - often use measures that do not discriminate between conditions, but these disorders may differ from one another in their social patterning across the lifecourse. The Twenty-07 Study includes the Hospital Anxiety and Depression Scale (HADS), which has sub-scales for each condition, allowing possible differences in patterning to be examined.

Objective: To investigate the age trajectories for anxiety and depression by social class.

Design and Setting: Prospective cohort study of 4150 men and women, living in Clydeside, aged 15, 35 and 55 at baseline in 1987/8 and interviewed at five-year intervals for 20 years. HADS scores were obtained at each of four follow-up visits and growth curve modelling was used to assess the relationship between HADS caseness, age, sex and baseline social class across 10629 measurement occasions from 3846 respondents. This sample is representative of those interviewed at baseline.

Results: There was a higher prevalence of anxiety than depression: $39.4 \%$ of the measurement occasions were defined as anxiety cases, $12.5 \%$ as depression cases, and $10.4 \%$ as cases for both disorders. There were significant non-linear age trajectories in caseness. The probability of anxiety caseness was relatively high in youth to middle-age and decreased with age thereafter. This age-related improvement was slower for those in manual, compared to nonmanual, classes, and this class difference was larger for females. The probability of depression caseness was low in youth, and increased with age with a steeper increase for those in manual classes than for those in non-manual classes. The probability of having both anxiety and depression exhibited an inverse U-shaped trajectory, peaking in middle age, with a class difference in the age gradient similar to that for depression. Sensitivity analyses indicated that these findings were robust to period and cohort effects as well as sample attrition. Conclusion: Anxiety and depression exhibit quite different trajectories across the lifecourse; the probability of anxiety reduces with age, whilst depression becomes more probable. There is a significant interaction between social class and age in both conditions, with those in manual classes having a slower reduction in anxiety and a faster increase in depression as they age than more affluent respondents. Future work should be sensitive therefore to how the social patterning of the two disorders differs across the lifecourse.

\section{SUICIDE IN ENGLAND AND WALES 1861-2005: A TIME TRENDS ANALYSIS}

K Thomas, D Gunnell. University of Bristol, Bristol, UK

\section{doi:10.1136/jech.2009.096701t}

Background: Suicide is amongst the three leading causes of death in 15-44-year-olds worldwide. A detailed assessment of secular trends in its incidence in England and Wales over the last 145 years has not previously been conducted; such an assessment may shed light on the potentially preventable factors and provide pointers concerning likely trends in suicide in the world's emerging economies

Aim: To investigate age-, sex- and method-specific trends in suicide from 1861 to 2005.

Methods: Suicide and population data were obtained from the ONS. Overall age-standardised rates using the European Standard
Population and age-, sex- and method-specific suicide rates were calculated for ages 15 and over.

Results: Rates in males (M) were consistently higher than females (F) throughout the 19th and 20th centuries, although the sex ratio fluctuated from $11 \mathrm{M}: 2 \mathrm{~F}$ in the 1880 s to $8 \mathrm{M}: 5 \mathrm{~F}$ in the $1960 \mathrm{~s}$. The highest male rates (28 per 100000 ) were recorded in the $1890 \mathrm{~s}$ Female rates peaked in the 1930s and 1960s (10 per 100 000). In both sexes the lowest recorded rates were in the 21st century. Suicide rates increased in all age groups in the 1930s, coinciding with the Great Depression. Over the period studied, rates fell by $87 \%$ in men aged $65+$, by $66 \%$ in women ages $65+$, but rose in younger men (aged 15-44). There was a rapid rise in the use of domestic gas as a method of suicide in both sexes following its introduction at the end of the 19th century. By the 1950s it had become the most commonly used method of suicide, before its use declined after the 1960s. Self poisoning also increased in popularity from the 1860s (5\% of suicides, mainly ingestion of hydrochloric, oxalic and carbolic acid) to the 1990s (22\% of suicides: mainly analgesics and antidepressants). The sex ratio was consistently lowest for suicides by poisoning and highest for suicides by hanging for most of the time period.

Conclusions: The rapid rise in gas suicide deaths in the 1920s highlights how quickly a new method of suicide can be established in a population when it is easily available. The increase in suicides during the Great Depression has implications in relation to the current economic crisis. The striking changes in suicide rates over time remain largely unexplained. Differences in the acceptability of various suicide methods are likely to account for the differences in sex ratios seen for hanging and poisoning.

\section{Cancer I}

\section{SEASONAL VARIATION IN BIRTH AND DIAGNOSIS OF CANCER IN CHILDREN AND YOUNG PEOPLE IN NORTHERN ENGLAND, 1968-2005}

${ }^{1} \mathrm{NO}$ Basta, ${ }^{1} \mathrm{PW}$ James, ${ }^{2} \mathrm{AW}$ Craft, 'RJO McNally. ${ }^{1}$ Institute of Health and Society, Newcastle University, Sir James Spence Institute, Royal Victoria Infirmary, Newcastle upon Tyne, UK; ${ }^{2}$ Northern Institute of Cancer Research, Newcastle University, Sir James Spence Institute, Royal Victoria Infirmary, Newcastle upon Tyne, UK

\section{doi:10.1136/jech.2009.096701u}

Objectives: To investigate seasonal variation in the incidence of cancer in children and young people, using population-based data. Setting: Northern England, UK.

Design: Data on all cases were extracted from the Northern Region Young Persons' Malignant Disease Registry (NRYPMDR). The NRYPMDR is a specialist registry that records cancer cases in children and young adults covering the counties of Northumberland, Tyne and Wear, Durham, Teesside, and Cumbria (excluding Barrow-in-Furness)

Participants: All cancer cases aged 0-24 diagnosed during the period 1968-2005 and registered by the NRYPMDR.

Methods: The following diagnostic groups were analysed: leukaemia (acute lymphoblastic, acute non-lymphocytic), lymphoma (Hodgkin, non-Hodgkin), central nervous system tumours (astrocytoma, primitive neuroectodermal tumours (PNETS)), sympathetic nervous system tumours, retinoblastoma, renal tumours, hepatoblastoma, bone tumours (osteosarcomas, Ewing sarcoma), soft tissue sarcoma, germ cell tumours (gonadal, non-gonadal) and carcinomas (thyroid, skin, malignant melanoma, breast, cervical). The chi-squared heterogeneity test was used to test for departure from the uniform distribution. Poisson regression analysis was used to fit sinusoidal (harmonic) models to the data, using month of birth and month of diagnosis, respectively, as covariates in separate models. Analyses were carried out separately by gender and age group (0-14, 1524 years) 
Results: There were a total of 5909 childhood cancer cases; 2959 aged 0-14 years (1659 males, 1300 females) and 2950 aged 1524 years (1590 males, 1358 females). For 0-14-year-old boys, there was statistically significant sinusoidal variation in month of birth for acute non-lymphocytic leukaemia ( $p=0.04$; peak in September) and astrocytoma $(p=0.03$; peak in October). Based on month of diagnosis, there was statistically significant sinusoidal variation in girls for all lymphomas ( $p=0.05$; peak in March) and Hodgkin lymphoma ( $p=0.005$; peak in January), and in boys for osteosarcoma ( $p=0.05$; peak in October). For 15-24-year-olds, there was significant heterogeneity for germ cell tumours $(p=0.04)$, cervical $(p=0.03)$ and female breast carcinoma $(p=0.05)$, based on month of birth, and PNETs $(p=0.05)$ and skin carcinoma $(p=0.05)$, based on month of diagnosis. Significant sinusoidal variation in month of birth for malignant melanoma in females $(p=0.03$; peak in March) and cervical carcinoma ( $p=0.03$; peak in October) was observed.

Conclusions: These findings suggest that seasonal environmental factors around the time of birth or time of diagnosis may be involved in the aetiology of specific diagnostic groups. Further research is needed to study possible aetiological mechanisms and factors. Putative agents include sunlight, pesticides, diet and infections.

\section{THE EPIDEMIOLOGY OF BONE CANCER DIAGNOSED IN 0-49- YEAR-OLDS IN NORTHERN ENGLAND, 1981-2002}

${ }^{1} \mathrm{RG}$ Feltbower, ${ }^{2} \mathrm{R}$ Eyre, ${ }^{2} \mathrm{PW}$ James, ${ }^{2} \mathrm{~K}$ Blakey, ${ }^{2} \mathrm{E}$ Mubwandarikwa, ${ }^{3,4} \mathrm{D}$ Forman, ${ }^{1}$ PA McKinney, ${ }^{2} \mathrm{MS}$ Pearce, ${ }^{2} \mathrm{RJO}$ McNally. ${ }^{1}$ Paediatric Epidemiology Group, Centre for Epidemiology and Biostatistics, University of Leeds, Leeds, UK; ${ }^{2}$ Institute of Health and Society, Newcastle University, Sir James Spence Institute, Royal Victoria Infirmary, Newcastle upon Tyne, UK; ${ }^{3}$ Northern and Yorkshire Cancer Registry and Information Service, Bexley Wing, St James's University Hospital, Leeds, UK; ${ }^{4}$ Cancer Epidemiology Group, Centre for Epidemiology and Biostatistics, University of Leeds, Leeds, UK

\section{doi:10.1136/jech.2009.096701v}

Aims: We aimed to describe incidence and survival patterns for bone cancers diagnosed during 1981-2002 in northern England among 0-39-year-olds.

Methods: 509 cases with a malignant bone tumour were included the analysis and classified using the International Classification of Diseases for Oncology, second edition. Incidence trends were analysed using Poisson regression. Survival rates were calculated using Kaplan-Meier estimation and differences in survival between diagnostic groups assessed using log-rank tests. Cox regression analysis was used to model the probability of survival in relation to age, gender and year of diagnosis.

Results: Overall incidence (per million person years) rates were 3.0 (95\% CI 2.6 to 3.4) for osteosarcoma, 2.1 (1.8 to 2.5) for Ewing sarcoma and 0.8 (0.6 to 1.0) for chondrosarcoma. Incidence of osteosarcoma increased significantly by an average annual rate of $2.6 \%(p=0.02)$ although there was no change in incidence for Ewing sarcoma or chondrosarcoma. Survival improved for Ewing sarcoma (hazard ratio (HR) per annum $0.97,95 \%$ CI 0.94 to 1.00), although patients aged $15-39$ years $(n=93)$ had worse overall survival than those aged $0-14(n=73)$ (HR 1.46, 95\% CI 0.98 to 2.17). There was no improvement in osteosarcoma survival (HR per annum $0.98,95 \%$ CI 0.95 to 1.01 ).

Conclusions: Our data suggested that incidence of osteosarcoma increased significantly in contrast to other bone tumours among 0 39-year-olds and previous findings from the UK and USA. Poorer survival in older Ewing sarcoma patients is consistent with previous studies and may be due to treatment, delays in diagnosis, metastatic disease, site and the stage of the tumour. The failure to improve survival for osteosarcoma patients needs further investigation.

\section{INCIDENCE OF CENTRAL NERVOUS SYSTEM TUMOURS WITH THE USE OF HORMONE REPLACEMENT THERAPY}

V Benson, K Pirie, J Green, G Reeves, V Beral, on behalf of the Million Women Study Collaborators Cancer Epidemiology Unit, University of Oxford, Oxford, UK

doi:10.1136/jech.2009.096701w

Background: Approximately 4300 people are diagnosed with a brain or other central nervous system (CNS) tumour annually in the UK. They have poor prognosis, yet little is known about risk factors of the tumours.

Objectives: To investigate the role of hormone replacement therapy (HRT) and the risk of brain and CNS tumours, specifically gliomas, meningiomas, and acoustic neuromas in post-menopausal women.

Design: Prospective cohort study.

Participants and Setting: 1.1 million post-menopausal women were recruited from breast screening clinics from 1996-2001 in England and Scotland and followed for incident tumours through NHS cancer registration. All CNS tumours and each of the tumour types (glioma, meningioma, and acoustic neuroma) were separate end points in a Cox proportional hazards model for investigation of various measures of HRT use.

Main Outcome Measure: Relative risk (RR) of incident CNS tumours (classified as malignant or benign) relating to the use of HRT, adjusting for age, socioeconomic status, region of residence, height, and body mass index.

Preliminary Results: A total of 1163 post-menopausal women with HRT information were diagnosed with a tumour of the brain or CNS; 517 tumours were classified as glioma, 280 as meningioma, and 113 as acoustic neuroma. Preliminary findings show that current users of HRT were more likely to develop a CNS tumour when compared to never users (RR $1.18,95 \%$ CI 1.03 to 1.35). Findings by type of HRT use and by the histological subtype of the tumours will be presented.

Conclusion: Current users of HRT are at a slightly increased risk of developing CNS tumours.

\section{INTERNATIONAL TRENDS IN PROSTATE CANCER INCIDENCE AND MORTALITY IN 20 COUNTRIES FROM 1980 TO 2002}

${ }^{1} \mathrm{AE}$ Carsin, ${ }^{2} \mathrm{E}$ Masuyer, ${ }^{1} \mathrm{~L}$ Sharp, ${ }^{2} \mathrm{P}$ Autier. ${ }^{1}$ National Cancer Registry, Cork, Ireland; ${ }^{2}$ International Agency for Research on Cancer, Lyon, France

doi:10.1136/jech.2009.096701x

Objective: Worldwide, since the 1980s, there have been major changes in prostate cancer detection and treatment with the availability of the prostate specific antigen (PSA) test and the introduction of hormone therapy and radical prostatectomy. To explore the effect of these advances on the burden of disease, we investigated time trends in prostate cancer incidence and mortality in 20 industrialised countries.

Data Source: Data on prostate cancer incidence during 1980 to 2002 was extracted from volumes 6 to 9 of Cancer Incidence in Five Continents. Mortality data from 1990 to 2002 were obtained from the WHO mortality database.

Methods: Age-standardised rates (ASR), based on the male population aged $\geqslant 50$, were calculated for each year in each country using the European Standard Population. Annual percentage change (APC) in incidence and mortality rates, and the points in time when trends changed, were estimated by fitting join point regression models using Join point software (3.1). Overall percentage change $(\mathrm{OPC})$ during the study period was defined as $\mathrm{OPC}=(1+\mathrm{APC})^{\text {number }}$ of years -1 . Trends for men aged $50-74$ and $\geqslant 75$ years were also analysed. 